\title{
Erratum to: Study on vulnerability matrices of masonry buildings of mainland of China
}

\author{
Sun Baitao ${ }^{1,2 \dagger}$ and Zhang Guixin ${ }^{1,2 \ddagger}$ \\ 1. Institute of Engineering Mechanics, China Earthquake Administration, Harbin 150080, China \\ 2. Key Laboratory of Earthquake Engineering and Engineering Vibration of China Earthquake Administration, Harbin 150080, China
}

In Vol. 17, No. 2

Locations:

Pages 251, 253, 255, 257 and 259: in the title and running title

Page 251, in the third and fifth lines of the Abstract

Page 251, in the twelfth line of the Footnote

Page 252, the right column, in the last line of the first paragraph

Page 253, the left column, in the sixth line of the third paragraph

Page 255, in the sixth line of the right column

Page 258, in the ninth line of the second paragraph of the Conclusions

Page 258, in the last line of the Acknowledgement

Page 259, in the first line of the right column

Remarks:

The word "of" should be added after the word "mainland".

Correspondence to: Zhang Guixin, Institute of Engineering

Mechanics, China Earthquake Administration, Harbin

150080, China

Tel: +8645186652954

E-mail: zgx@iem.cn

†Professor; ${ }^{\ddagger}$ Assistant Professor 